\title{
Emergency airway management in a case of lingual haematoma
}

\author{
R J Shaw, G W McNaughton
}

\begin{abstract}
A previously unreported cause of acute tongue swelling is presented and the airway issues discussed. Cases with different aetiology have been sporadically published however the consequent, and sometimes fatal, airway obstructions have been dealt with somewhat variably. The aetiogy of acute tongue swelling and modern emergency airway algorithms are discussed with reference to the literature. (Emerg Med f 2001;18:408-409)
\end{abstract}

Keywords: emergency airway management; lingual haematoma

\section{Case report}

A 50 year old alcoholic man was admitted to the accident and emergency department in a collapsed state. Primary survey demonstrated obstructed upper airway noises, presence of blood in and around the mouth and massive tongue swelling. Further examination revealed lingual and sublingual haematoma sufficient to prop the mouth open (fig 1). After sitting the patient forwards the airway was immediately improved with simultaneous improvement in conscious level. The patient was tachycardic, tremulous and initially uncooperative, giving no useful history. Attending relatives gave a history that he had stopped drinking four days previously, following a daily alcohol habit of 30 units. The working diagnosis was alcohol withdrawal seizures complicated by lingual haematoma secondary to tongue biting. Anaesthetic and ENT opinions regarding airway management and admission were obtained.

Medical management was instituted with intravenous corticosteroids in an attempt to limit any further lingual oedema (hydrocortisone $250 \mathrm{mg}$ four times a day) and carefully titrated benzodiazepines to prevent further seizure activity (diazepam 1-2 mg increments intravenously). Investigations revealed thrombocytopenia (platelets $40 \times 10^{9} / 1$ ), coagulopathy (prothrombin time $24 \mathrm{~s}$, activated partial

and Maxillofacial

Surgery, University Hospital Aintree,

Liverpool, UK

R J Shaw

Accident and

Emergency Medicine

Department, Royal

Alexandra Hospital,

Corsebar Road,

Paisley, Renfrewshire

PA2 9PN, Scotland

G W McNaughton

Correspondence to:

Dr McNaughton

(gordon.mcnaughton@)

rah.scot.nhs.uk)

Accepted for publication 11 June 2001

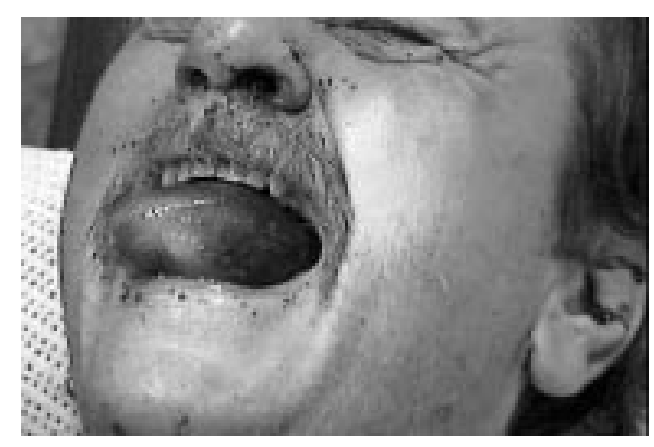

Figure 1 Lingual enlargement at presentation in the accident and emergency department.
Table 1 The Lemon Law

\begin{tabular}{lll}
\hline Mnemonic: & Findings in case: \\
\hline & & \\
$\mathrm{L}$ & Look carefully & Trauma/blood/moustache \\
$\mathrm{E}$ & Evaluate 3-3-2 rule & (no problem) \\
$\mathrm{M}$ & Mallampati† & Grade IV \\
$\mathrm{O}$ & Obstruction & Massive tongue swelling \\
$\mathrm{N}$ & Neck movement & (no problem) \\
\hline
\end{tabular}

*Evalaution of neck anatomy in terms of finger's breadth distances 3 fingers: mouth opening, 3 fingers: chin to hyoid, 2 fingers: hyoid to thyroid notc. †Mallampati grade of intubation difficulty according to structures visible on maximum mouth opening: I entire opharynx including base of tonsil, II uvula tip and Fauces, III soft palate and base of uvula, IV hard palate only.

thromboplastin time $40 \mathrm{~s}$, fibrinogen $120 \mathrm{mg} / \mathrm{dl}$ ) and alcoholic liver disease (bilirubin $95 \mu \mathrm{mol} / \mathrm{l}$, $\gamma$-glutamyltransferase 998 IU/l, alkaline phosphatase $46 \mathrm{IU} / 1)$. Coagulation was rapidly normalised with fresh frozen plasma and platelet transfusion. The decision not to create a surgical airway was made only after a period of active and continuous observation. A little further swelling was well tolerated and the patient made a good recovery to be discharged eight days later.

\section{Discussion}

Acute enlargement of the tongue is rare but a recognised airway hazard that has been classified by Renehan and Morton. ${ }^{1}$ Catagories include haematoma resulting from trauma, vascular anomalies or coagulopathy; and also oedema, infarction and infection. This is the first reported case of its type, demonstrating two subclasses of aetiology (trauma and coagulopathy) both mediated by chronic alcohol misuse. Trauma caused by road traffic accident, ${ }^{23}$ dental surgery ${ }^{4}$ and tongue biting in eclamptic seizure ${ }^{5}$ have been described. Warfarin appears in several cases as a factor in spontaneous lingual haematoma ${ }^{6}$; additionally streptokinase $^{7}$ and haemophilia ${ }^{8}$ have been implicated in isolated reports. The value of corticosteroids has been described in previous cases although remains of unproven benefit. ${ }^{16}$

Progressive lingual and sublingual swelling displaces the tongue posteriorly and cephalad eventually producing dysphonia, drooling, dyspnoea and finally stridor heralding upper airway obstruction. ${ }^{4}$ In the presence of these features, it is axiomatic that a definitive airway must be established early in this sequence, the first step being assessment for ease of intubation. ${ }^{9}$ An airway may be assessed according to the "Lemon Law" established by the National Emergency Airway Management Course (USA) (table 1).

The airway was assessed as difficult and the chances of successful intubation via the orotracheal route were thought poor. Consequently 
the management proceeded along the lines of the difficult airway protocol, ${ }^{910}$ consideration being given in turn to several alternatives.

The first method suggested by the protocol, blind nasal tube intubation, was contraindicated by the presence of upper airway obstruction, coagulopathy and possible neck haematoma. Rapid sequence induction was ruled out because of predictable problems with bag and mask ventilation - that is, loss of airway when supine and presence of facial hair. Fibreoptic intubation via the nasal route under local anaesthetic and sedation was then considered, although also relatively contraindicated by bleeding in, and obstruction of, the upper airway. Consequently a surgical airway was planned and an emergency cricothyroidotomy set prepared as a precaution. Local anaesthetic open tracheostomy, a formidable prospect in an upright, coagulopathic and combative patient, was considered and specialist opinion obtained. The decision not to proceed was influenced by stability of the patient, expected clinical course (once coagulation corrected) and admission to a high dependency unit permitting continuous observation. This case illustrates several of the dilemmas encountered when following established airway algorithms.

Review of the literature shows a total of 21 cases $^{1-8}$ of airway obstruction attibutable to tongue swelling, only four of which were managed by active observation (one with fatal consequence). Of the 17 cases with definitive airways, 10 were surgical airways with two cricothyroidotomies and eight tracheostomies. Two of the remaining patients underwent blind nasal tube intubation, two had fibreoptic intubation and in the other four cases the method of intubation was not specified.

While the immediate value of simple airway manoeuvres (such as sitting forwards, nasopharyngeal tube) are not in question, a definitive airway will be required in most cases. Management of acute tongue swelling by active observation should thus be approached with extreme caution. Both current protocol and case history suggest that a surgical airway is often indicated. In every case, urgent consultation with senior anaesthetic and surgical staff is advised.

We are grateful to Ms Aileen White, consultant otolaryngologist for her advice in preparation of this report and to whose care the for her advice in preparat
patient was referred.

1 Renehan A, Morton M. Acute enlargement of the tongue. Br F Oral Maxillofac Surg 1993;31:321-4.

2 Hing NR, Bowler MD, Byth PL, et al. Lingual haemotoma leading to upper airway obstruction. Br $\mathcal{F}$ Oral Maxillofac Surg 1985;23:322-5

3 Chase CR, Herbert JC, Rarnham JE. Post-traumatic upper airway obstruction secondary to a lingual artery haematoma. $\mathcal{F}$ Trauma 1987;27:953-4.

4 Kattan B, Snyder HS. Lingual artery haematoma resulting in upper airway obstruction. F Emerg Med 1991;9:421-4.

5 Saah D, Elidan J, Braverman I, et al. Traumatic macroglossia. Ann Otol Rhinol Laryngol 1993;102:729-30.

6 Cohen AF, Warman SP. Upper airway obstruction secondary to warfarin induced sublingual haematoma. Arch Otolaryngol Head Neck Surg 1989;115:718-20.

7 Williams PJ, Jani P, McGlashan J. Lingual haematoma following treatment with streptokinase and heparin; following treatment with streptokinase and hepart

8 Takeuchi M, Shikimori M, Kaneda T. Life-threatening sublingual haematoma in a severely hemophilic patient with factor VIII inhibitor. 7 Oral Maxillofac Surg 1986;44:401-3.

9 Walls RM, ed. Manual of emergency airway management. (Companion Manual to the National Emergency Airway Management Course 2000). Philadelphia: Lippincott Williams and Wilkins, 2000.

10 American Society of Anaesthesiologists Task Force on the Difficult Airway: ASH difficult airway algorithm. Anaesthesiology 1993;58:597. 